\title{
Análise da viabilidade econômica da piscicultura em tanque escavado: estudo de caso no município de Tomé-Açu
}

A piscicultura, processo pelo qual se realiza a criação de peixes, podendo proceder em diferentes estruturas e modalidades de cultivo, tem ganhado cada vez mais espaço no mercado, isso se dá em função da relevância econômica que essa atividade vem demonstrando. Em consequência a esse fator, surge o interesse dos pequenos e médios produtores, uma vez que esse sistema não requer grandes extensões, nem altos investimentos para sua implantação, favorecendo boa rentabilidade e taxa de retorno. Assim, a presente investigação teve como objetivo realizar um estudo para atestar a viabilidade econômica da piscicultura na modalidade de tanque escavado de um produtor no Município de Tomé-Açu, atuando na criação do Curimatã (Prochilodus sp.) e o Tambatinga, o qual resulta do cruzamento entre a fêmea do tambaqui (Colossoma macropomum) e o macho da pirapitinga (Piaractus brachypomus), em cultivo intensivo. Os procedimentos metodológicos utilizados no estudo se deram a partir de uma pesquisa bibliográfica de caráter exploratório onde buscou-se encontrar fontes a respeito do tema proposto, sendo ainda de natureza quantitativa, haja vista, que se realizou a análise dos dados numéricos através dos procedimentos estatísticos. Em vista disto, para atestar a viabilidade dos fluxos de caixa elaborados para produção no município, foram calculados os indicadores do VPL (Valor Pressente Líquido), IBC (Índice Benefício Custo), TIR (Taxa Interna de Retorno), PB (Payback) e P.E (Ponto de Equilíbrio), submetidos a diferentes taxas de desconto de fontes de financiamentos existentes na região.

Palavras-chave: Custo de Produção; Indicadores de Viabilidade; Piscicultura; Tanque Escavado.

\section{Economic viability analysis of pisciculture in excavated tank: case study in the municipality of Tomé-Açu}

\begin{abstract}
Fish farming the process by which fish farming takes place, being able to proceed in different structures and modalities of cultivation, has gained more and more space in the market, this is due to the economic relevance that this activity has been demonstrating. As a result of this factor, the interest of small and medium producers arises, since this system does not require large extensions, nor high investments for its implementation, favoring good profitability and rate of return. Thus, the present investigation aimed to carry out a study to attest to the economic viability of fish farming in the modality of tank excavated from a producer in the municipality of Tomé-Açu, acting in the creation of Curimatã (Prochilodus sp.) and Tambatinga, which results from the crossing between the female of tambaqu (Colossoma macropomum) and the male of the pirapitinga (Piaractus brachypomus), in intensive cultivation. The methodological procedures used in the study were based on exploratory bibliographic research where we sought to find sources on the proposed theme, being also quantitative in nature, given that the numerical data were analysed through statistical procedures. In view of this, to attest to the feasibility of cash flows prepared for production in the municipality, the indicators of NPV (Net Pressure Value), IBC (Cost Benefit Index), IRR (Internal Rate of Return), PB (Payback) and P.E (Equilibrium Point) were calculated, submitted to different discount rates from existing funding sources in the region.
\end{abstract}

Keywords: Production Cost; Feasibility Indicators; Fish Farming; Tank Dug.

Topic: Desenvolvimento, Sustentabilidade e Meio Ambiente

Reviewed anonymously in the process of blind peer.

João Paulo Borges de Loureiro (iD

Universidade Federal Rural da Amazônia, Brasil

http://lattes.cnpq.br/0417040128680889

http://orcid.org/0000-0001-8586-8201

joaopaulo_loureiro@hotmail.com

Leidiane Silva dos Santos (iD

Universidade Federal Rural da Amazônia, Brasil

http://lattes.cnpq.br/7848261200734947

http://orcid.org/0000-0002-7660-8325

leidiane_santos16@outlook.com

Jamylle Telino Nogueira Rocha

Universidade Federal Rural da Amazônia, Brasil

http://lattes.cnpq.br/5240076714405005

http://orcid.org/0000-0002-0045-4534

mylletelino@gmail.com
Received: 01/04/2021

Approved: 28/04/2021

Taiete Pessoa de Oliveira

Universidade Federal Rural da Amazônia, Brasil

http://lattes.cnpq.br/8461214737222021

http://orcid.org/0000-0002-2022-7232

laieteoliveira@gmail.com

Luciane Cristina Costa Soares

Universidade Federal Rural da Amazônia, Brasil

http://lattes.cnpq.br/1411520200496551

http://orcid.org/0000-0003-4652-550X

crisluani@gmail.com
Referencing this:

LOUREIRO, J. P. B.; SANTOS, L. S.; ROCHA, J. T. N.; SOARES, L. C. C.. Análise da viabilidade econômica da piscicultura em tanque escavado: estudo de caso no município de Tomé-Açu. Revista Ibero Americana de Ciências Ambientais, v.12, n.4, p.590-597, 2021. DOI: http://doi.org/10.6008/CBPC2179-6858.2021.004.0045

DOI: 10.6008/CBPC2179-6858.2021.004.0045 


\section{INTRODUÇÃO}

A piscicultura é definida como a prática de produção de alevinos ou peixes em ambientes denominados como açude, alagado, reservatório, viveiro, ou tanque, representada por uma área formada pela contenção de água, havendo o controle de entrada e saída desse fluxo (IAP - Instituto Ambiental do Paraná).

Segundo Schulter et al. (2017) são desafios a serem enfrentados pelos países nas próximas décadas, a segurança alimentar e o crescimento populacional no mundo, pois haverá a necessidade de produzir mais alimentos. Alimentos como carne bovina, aves, suínos e pescados, para garantir que não falte alimentos na mesa dos consumidores. O pescado, por possuir componentes nutricionais importantes para os seres humanos, como o ômega 3, por ser de criação relativamente rápida e fácil, vem ganhando destaque como atividade de subsistência e em sistemas de criação intensivo.

Segundo Martins et al. (2001) com o passar do tempo percebeu-se uma grande migração de alguns produtores de culturas rurais para outras atividades rurais, dentre elas o setor piscícola que vem atraindo parte desses produtores e ainda de alguns empresários agrícolas. Isso se dá em função da relevância econômica que essa atividade vem demonstrando o que consequentemente desperta o interesse dos pequenos e médios produtores, haja vista que esse sistema não exige grandes extensões, tampouco altos investimentos para sua implantação, potencializando boa rentabilidade e taxa de retorno.

Conforme Vilela et al. (2012), dentre as atividades agropecuárias brasileiras o setor piscícola vem se destacando como uma das atividades de grande importância econômica, todavia, são poucas as informações de caráter técnico e econômico que possam auxiliar na execução de um planejamento. Pare se ter mais segurança na implantação desse sistema se faz necessário a existência de indicadores econômicos que demonstrem o sucesso ou não do negócio, o que consequentemente resultaria na evolução da atividade.

Analisar a viabilidade econômica de um determinado projeto, expressa a ideia de mensurar e estudar as expectativas de desempenho financeiro do produto e serviço, objetivando atestar se o projeto de implantação será economicamente viável ou não, de tal forma a atestar se este resultará em resultados positivos ou negativos para o empreendedor.

Neves (2010), afirma que estudar a viabilidade econômica de um projeto ou empreendimento é a prática de analisar os ativos humanos, recursos financeiros, bens permanentes e materiais, evidenciando a competência do empreendimento em conceber lucro, além de atestar a capacidade de recuperação do capital investido inicialmente na implantação do projeto. Para se atestar a viabilidade econômica, BordeauxRêgo et al. (2013), declara que deve ser considerado o tempo de vida útil do projeto, que de acordo o autor é "a vida econômica ou obsolescência, respectivamente, dos equipamentos iniciais do projeto ou do bem ou serviço produzido".

Assaf Neto et al. (2009) corroboram da mesma idealização ao afirmarem que as tomadas de decisões englobam todo um processo de assimilação das alternativas e posteriormente na escolha daquela que viabilize maior índice de rentabilidade econômica em um período futuro, a fim de garantir a sua perpetuidade 
e valorização. "As decisões de investimentos são atraentes quando os investimentos realizados criam valor aos proprietários de capital, ou seja, quando a taxa de retorno exigida excede ao retorno mínimo esperado da alternativa de investimento" (ASSAF NETO et al., 2009).

Em vista disso, viu-se a necessidade de analisar a viabilidade econômica da piscicultura na criação de peixes utilizando-se da modalidade tanque escavado, em um empreendimento de um produtor independente no município de Tomé-Açu, localizado no estado do Pará.

A procura por alimentos mais nutritivos, que contenham baixas proporções de gordura e caloria, e com alto índice de proteínas, vitaminas e minerais, leva a população a buscar alimentos provenientes do mar como peixes, mariscos e crustáceos, os quais proporcionam maior bem-estar às pessoas. Em vista disso, na atualidade o consumo do peixe tem sido crescente, uma vez que a população, em busca de uma alimentação mais saudável, vem percebendo os benefícios que o consumo desse produto traz para a saúde, quando comparados com a carne vermelha.

No município de Tomé-Açu este cenário não é diferente, pois percebe-se uma alta demanda e consumo desse produto. Dessa forma, o presente trabalho originou-se com a finalidade de analisar como ocorre o processo da criação de peixes no município e qual a sua viabilidade econômica para os produtores da região.

Em se tratando da implantação de um negócio, Vilela et al. (2012) enfatiza a necessidade de se executar um bom planejamento, controlando as despesas e receitas resultantes da atividade piscícola, utilizando-se de técnicas que permitam quantificar os custos e a rentabilidade do empreendimento.

Levando em consideração o exposto acima, o presente trabalho teve como objetivo realizar um estudo para atestar a viabilidade econômica da produção piscícola em tanque escavado no município de Tomé-Açu, onde será possível atestar se esse sistema de produção pode ser uma alternativa de geração de renda ao produtor independente no município, sendo que ao final da pesquisa foi possível responder a seguinte questão: Caso exista, qual o grau de rentabilidade da implantação da piscicultura, na modalidade tanque escavado, como meio de produção de peixes no município estudado?

\section{MATERIAIS E MÉTODOS}

A metodologia é uma somatória de procedimentos realizados, isto é, etapas percorridas para execução de uma pesquisa ou estudo. Desta maneira a elaboração do trabalho teve seu início a partir de uma pesquisa bibliográfica, que de acordo com Gil (2002) refere-se a instrumentos já elaborados, formado essencialmente por artigos, livros e demais publicações científicas, em que no trabalho em questão buscouse encontrar nesses materiais fontes a respeito do tema apresentado, sendo estes, a piscicultura, custos de produção e indicadores de viabilidade. A pesquisa em pauta é de cunho exploratório, tendo em vista que pesquisas deste caráter buscam oportunizar a compreensão do problema de tal forma que ele se torne nítido ou possibilitando ainda a construção de hipóteses, sendo ainda de natureza quantitativa onde realiza-se a análise dos dados numéricos através de procedimentos estatísticos, possibilitando a geração de tabelas de forma manual ou por meio de ferramentas como softwares com o auxílio do computador (GIL, 2002). 
Em seguida, foram realizadas as visitas junto ao produtor independente, localizado no município de Tomé-Açu, onde foram aplicadas planilhas de implantação e manutenção, obtendo-se os dados para a geração do fluxo de caixa. Posteriormente foi utilizado o software Excel, versão 2016, para tabulação dos dados, gerando assim o fluxo de caixa da piscicultura, considerando um horizonte para análise produtiva de dez anos, com investimento integral aplicado no ano zero.

Para verificar se o sistema é ou não viável, foi aplicado os indicadores do VPL, IBC, TIR, Payback e P.E, os quais foram submetidos a diferentes taxas de desconto de fontes de financiamentos existentes na região, sendo os indicadores calculados através das seguintes fórmulas matemáticas:

Valor Presente Líquido (VPL):

$$
V P L=F C 0+\frac{F C 1}{(1+i)^{1}}+\frac{F C 2}{(1+i)^{2}}+\ldots \frac{F C n}{(1+i)^{n}}
$$

Onde:

$\mathrm{VPL}=$ Valor presente líquido $\mathrm{FCO}=$ Ano zero de investimento

FC1 = Ano 1 de investimento $\mathrm{n}=$ Múltiplos indefinido de um fator

$$
\mathrm{i}=\text { Taxa }
$$

Taxa Interna de Retorno (TIR):

$$
T I R=\left(\frac{L . L}{I}-1\right) \times 100
$$

Onde: TIR = Taxa Interna de Retorna L.L = Lucro Líquido I = Capital Inicial

Índice Benefício Custo (IBC):

$$
I B C=\frac{\sum\left\lfloor C F_{j}\right\rfloor /(1+i)^{j}}{C F_{0}}
$$

Onde: $\mathrm{IBC}=$ Índice Benefício/Custo $\Sigma=$ Somatória $\mathrm{CFj}=$ Fluxo de Caixa

Payback (PB):

Ponto de Equilíbrio (PE): 


$$
Q=\frac{C F}{(P-C V m e)}
$$

\section{Estruturação e caracterização do empreendimento}

O estudo de caso foi realizado em um empreendimento voltado ao segmento piscícola na modalidade tanque escavado em cultivo intensivo, localizado na Rodovia PA 140, Tabocal Ramal - Km 6, de um produtor independente. A empresa possui alguns pontos que a fazem obter vantagem competitiva com relação aos seus concorrentes, como por exemplo, o fato de dispor de um alimento saudável que vem ganhando cada vez mais seu espaço no mercado, em função da busca da população por uma alimentação mais saudável, além de fornecer preço atrativo. 0 empreendimento é considerado de pequeno porte e tem como principal objetivo fornecer peixes de boa qualidade, satisfazendo as expectativas dos clientes.

A implantação das instalações do projeto já atinge 1 ano. A área total do empreendimento compreende 9 hectares, sendo dividido em duas especialidades: piscicultura, onde há 6 tanques escavados, envolvendo uma extensão de 30/20 metros cada, destinados à criação de peixes; e agricultura, na qual são cultivadas algumas espécies de frutas como, limão, laranja, açaí, dentre outras. Um ponto positivo observado refere-se ao portfólio que é fornecido pelo produtor, o qual oferta dentre as espécies de maior demanda na região, o Tambatinga, o qual é decorrente do cruzamento gerido entre a fêmea do tambaqui (Colossoma macropomum) e o macho da pirapitinga (Piaractus brachypomus) e o Curimatã (Prochilodus sp.), visando ainda futuramente agregar a esse portfólio, o Pirarucu (Arapaima gigas). As atividades de manejo são realizadas pelo produtor em conjunto com apenas um colaborador.

\section{RESULTADOS E DISCUSSÃO}

Para fins de cálculo dos indicadores de viabilidade considerou-se um horizonte produtivo de 10 (dez) anos e ainda, a não existência de produção no Ano 0 das atividades, com isso o valor inicial do Fluxo de Caixa Líquido (FCL) corresponde ao primeiro investimento da produção, sendo o valor inicial investido pelo produtor de pequeno porte do Município de Tomé-Açu o equivalente a $\mathrm{R} \$ 25.900,00$. A estrutura da propriedade compreende um total de 9 hectares, onde $1 / 3$ desse total, ou seja, 3 hectares são destinados ao cultivo piscícola, o qual é composto por 6 tanques escavados com extensão de 30/20 metros em cultivo intensivo, tendo assim uma capacidade de produção de 2.850 peixes por ano, considerando uma taxa de mortalidade de $5 \%$.

Nos anos posteriores, a atividade aponta uma receita de $\mathrm{R} \$ 39.900,00$, tendo em vista que a produção anual equivale a 2.850 peixes, pesando em média $2 \mathrm{Kg} /$ unid., os quais são distribuídos nos pontos de revenda à $R \$ 7,00 / \mathrm{Kg}$. Sabendo-se que é gerado um custo de manutenção de $\mathrm{R} \$ 1$ 19.442,00, obtêm-se um 
FCL positivo de $\mathrm{R} \$ 20.458,00$ na produção piscícola, considerando o Ano 1. É importante salientar que a cada 2 anos o produtor realiza o processo de calagem dos tanques, procedendo primeiramente com a drenagem da água, para então suceder com a adubação da terra, aplicando o calcário, a fim de realizar a correção do solo, com o objetivo de equilibrar o pH e a alcalinidade do solo e da água, ressalta-se também que no Ano 5 haverá um custo com o implemento de uma nova rede de pesca. De acordo às informações, observa-se, portanto, uma variação anual dos custos de manutenção, influenciando diretamente no FCL, como mostra a tabela 1.

Tabela 1: Fluxo de caixa líquido e acumulado do sistema de produção piscícola no município de Tomé-Açu.

\begin{tabular}{|l|l|l|l|l|}
\hline & Custos & Receita & $\mathrm{FCL}$ & FC Acumulado \\
\hline Ano 0 & $\mathrm{R} \$ 25.900,00$ & $\mathrm{R} \$-$ & $-\mathrm{R} \$ 25.900,00$ & $-\mathrm{R} \$ 25.900,00$ \\
\hline Ano 1 & $\mathrm{R} \$ 19.442,00$ & $\mathrm{R} \$ 39.900,00$ & $\mathrm{R} \$ 20.458,00$ & $-\mathrm{R} \$ 5.442,00$ \\
\hline Ano 2 & $\mathrm{R} \$ 19.862,00$ & $\mathrm{R} \$ 39.900,00$ & $\mathrm{R} \$ 20.038,00$ & $\mathrm{R} \$ 14.596,00$ \\
\hline Ano 3 & $\mathrm{R} \$ 19.442,00$ & $\mathrm{R} \$ 39.900,00$ & $\mathrm{R} \$ 20.458,00$ & $\mathrm{R} 35.054,00$ \\
\hline Ano 4 & $\mathrm{R} \$ 19.862,00$ & $\mathrm{R} \$ 39.900,00$ & $\mathrm{R} \$ 20.038,00$ & $\mathrm{R} \$ 55.092,00$ \\
\hline Ano 5 & $\mathrm{R} \$ 20.742,00$ & $\mathrm{R} \$ 39.900,00$ & $\mathrm{R} \$ 19.158,00$ & $\mathrm{R} \$ 74.250,00$ \\
\hline Ano 6 & $\mathrm{R} \$ 19.862,00$ & $\mathrm{R} \$ 39.900,00$ & $\mathrm{R} \$ 20.038,00$ & $\mathrm{R} \$ 94.288,00$ \\
\hline Ano 7 & $\mathrm{R} \$ 19.442,00$ & $\mathrm{R} \$ 39.900,00$ & $\mathrm{R} \$ 20.458,00$ & $\mathrm{R} \$ 114.746,00$ \\
\hline Ano 8 & $\mathrm{R} \$ 19.862,00$ & $\mathrm{R} \$ 39.900,00$ & $\mathrm{R} \$ 20.038,00$ & $\mathrm{R} \$ 134.784,00$ \\
\hline Ano 9 & $\mathrm{R} \$ 19.442,00$ & $\mathrm{R} \$ 39.900,00$ & $\mathrm{R} \$ 20.458,00$ & $\mathrm{R} \$ 155.242,00$ \\
\hline
\end{tabular}

O cálculo do Valor Presente Líquido (VPL) ratifica a capacidade de o empreendimento suportar taxas de desconto, onde no estudo apresentado foi utilizado o Pronaf B (0,5\%) e o Pronaf Mais Alimentos, sendo este analisado frente à duas diferentes taxas (2,5\% e 5,5\%). Desta forma, analisando pelo âmbito financeiro, nota-se que a atividade piscícola suportaria facilmente taxas de juros de $0,5 \%$ à $10 \%$ a.a., sendo que esse fato inicial é consolidado através dos cálculos da Taxa Interna de Retorno (TIR) e do Índice Benefício Custo (IBC), onde o primeiro indicador demonstra que o empreendimento têm capacidade de suportar taxas de desconto de até $77,74 \%$ a.a., e o segundo indicador, por sua vez, demonstra um retorno de $R \$ 6,82$ com a taxa mais baixa à $R \$ 4,48$ com a taxa mais alta, para cada real investido. Os resultados ainda apontam um Payback de 2 anos e 3 meses, isto é, levando em consideração as instalações piscícolas em um horizonte de 10 anos, o produtor irá recuperar o investimento inicial no período de 2 anos e 3 meses iniciais do projeto, conforme apresentado no quadro 1.

Quadro 1: Resultado dos indicadores de viabilidade e Payback do sistema de produção piscícola em Tomé-Açu.

\begin{tabular}{|lllll|}
\hline & Pronaf B & PMA & PMA & Outras fontes \\
\hline Indicador & $0,5 \%$ & $2,5 \%$ & $5,5 \%$ & $10,0 \%$ \\
VPL & $\mathrm{R} \$ 150.795,38$ & $\mathrm{R} \$ 134.531,82$ & $\mathrm{R} \$ 114.038,68$ & $\mathrm{R} \$ 90.043,93$ \\
IBC & $\mathrm{R} \$ 6,82$ & $\mathrm{R} \$ 6,19$ & $\mathrm{R} \$ 5,40$ & $\mathrm{R} \$ 4,48$ \\
TIR & $77,74 \%$ & $77,74 \%$ & $77,74 \%$ & $77,74 \%$ \\
P.B & $2 \mathrm{~A} \mathrm{e} \mathrm{3M}$ & $2 \mathrm{~A} \mathrm{e} 3 \mathrm{M}$ & $2 \mathrm{~A} \mathrm{e} 3 \mathrm{M}$ & $2 \mathrm{~A} \mathrm{e} 3 \mathrm{M}$ \\
P.E & $2.825 \mathrm{Kg} /$ ano & $2.825 \mathrm{Kg} /$ ano & $2.825 \mathrm{Kg} / \mathrm{ano}$ & $2.825 \mathrm{Kg} /$ ano \\
\hline
\end{tabular}

O Ponto de Equilíbrio (PE) demonstra qual o nível de produção necessário para que o empreendimento consiga liquidar os custos totais aferidos durante o processo produtivo. Nesse ponto, ressalta-se que os custos totais equivalem $\mathrm{R} \$ 1$ 19.862,00 (valor correspondente ao Ano 2, uma vez que a cada 2 anos realiza-se a calagem dos tanques conforme citado anteriormente), sendo que $R \$ 10.548,00$ que compõem esse valor são referentes aos custos variáveis, e $\mathrm{R} \$ \mathbf{9 . 3 1 4 , 0 0}$ integram os custos fixos. Na tabela 
abaixo estão descritos, de forma detalhada todos os custos que geraram esse montante que está descrito na Quadro 2.

Quadro 2: Custos Fixos e Custos Variáveis do projeto.

\begin{tabular}{|c|c|c|c|}
\hline Custo Fixo (CF) & Valor & Custo Variável (CV) & Valor \\
\hline Mão-de-obra (Manejo) & $\mathrm{R} \$ 6.000,00$ & Animais (Alevinos) & $\mathrm{R} \$ 900,00$ \\
\hline Depreciação & $\mathrm{R} \$ 1.760,00$ & Ração Inicial & $\mathrm{R} \$ 468,00$ \\
\hline \multirow[t]{4}{*}{ Custo de Oportunidade } & $\mathrm{R} \$ 1.554,00$ & Ração de crescimento & $\mathrm{R} \$ 1.632,00$ \\
\hline & & Ração de engorda final & $\mathrm{R} \$ 1.728,00$ \\
\hline & & Gasolina (bomba D'água) & $R \$ 5.400,00$ \\
\hline & & Calagem dos tanques & $\mathrm{R} \$ 420,00$ \\
\hline Total (CF) & $\mathrm{R} \$ 9.314,00$ & Total (CV) & $\mathrm{R} \$ 10.548,00$ \\
\hline
\end{tabular}

À vista disso, a tabulação demonstrou um nível de produção total que corresponde a 2.850 peixes/ano, o que equivale a $5.700 \mathrm{Kg} / \mathrm{ano}$, considerando que o peso médio unitário é igual a $2 \mathrm{Kg}$, assim sendo o estudo constatou um PE de $2.825 \mathrm{Kg} / \mathrm{ano}$, correspondente à 49,56\% da capacidade produtiva anual, sabendo-se que os custos totais equivalem a $\mathrm{R} \$ 1$ 19.862,00, o valor do PE encontrado resulta em uma equivalência entre ambos. Logo, sendo a capacidade produtiva total igual a $5.700 \mathrm{Kg} / \mathrm{ano}$ e um PE de 2.825 $\mathrm{Kg} / \mathrm{ano}$, constata-se que $2.875 \mathrm{Kg} /$ ano são responsáveis pela geração dos lucros, conforme demonstrado no gráfico 1.

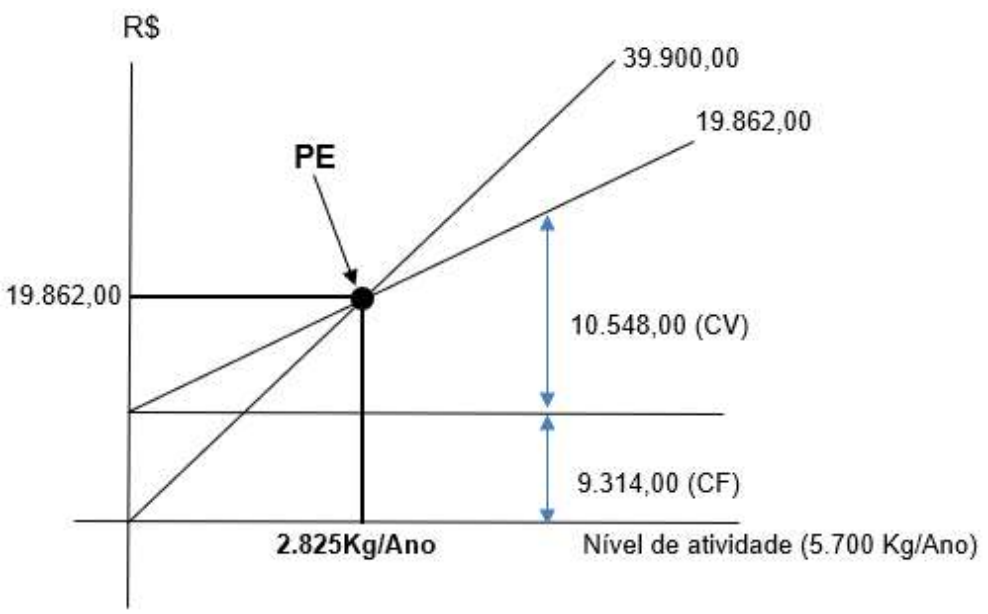

Gráfico 1: Ponto de equilíbrio (PE) do sistema de produção piscícola em Tomé-Açu.

Em complemento, para demonstrar o PE identificado, primeiramente encontra-se o Custo Total Médio - Ctme, através da divisão do Custo Total Anual - CT pela Quantidade Produzida por ano (R\$ 19.862,00/2.850 unid.), em seguida, encontra-se a Margem de Contribuição Unitária - MCU, onde subtrai-se o Preço recebido pelo Produtor (é recebido em média $R \$ 14,00$ por peixe) do Ctme (R\$ 14,00 - R\$ 6,97), e por fim, para constatar o PE, divide-se o CT pelo MCU (R\$19.862,00/R\$ 7,03), conforme a Quadro 3.

Quadro 3: Ponto de Equilíbrio (PE) e Margem de Contribuição Unitária.

\begin{tabular}{|ll|}
\hline Ponto de Equilíbrio Corrigido & $\mathbf{2 . 8 2 5} \mathbf{K g} /$ Peixe/Ano \\
\hline CT (Custo Total Anual) & $\mathrm{R} \$ 19.862,00$ \\
Ctme (Custo Total Médio) & $\mathrm{R} \$ 6,97$ \\
$\mathrm{P}$ (Preço recebido pelo Produtor) & $\mathrm{R} \$ 14,00$ \\
MCU (Margem de Contribuição Unitária) & $\mathrm{R} \$ 7,03$ \\
P.E (Ponto de Equilíbrio) & $2.824,97$ \\
Quantidade Produzida por ano & 5.700 \\
\hline
\end{tabular}




\section{CONCLUSÕES}

Fundamentado no propósito de explanar de forma teórica os procedimentos realizados na implantação e manutenção de instalações piscícolas na modalidade tanque escavado, embasando-se principalmente em conceitos e fundamentações teóricas voltados à custo de produção, onde considerou-se indicadores de viabilidade como: VPL, IBC, TIR, Payback e P.E, o presente trabalho resultou na comprovação da viabilidade econômica da criação de peixes utilizando-se a modalidade de tanque escavado de um produtor independente no Município de Tomé-Açu, ratificando a efetividade desse segmento da agricultura no município estudado.

Diante a afirmação supracitada, concluiu-se que a Piscicultura apresenta um grau satisfatório de rentabilidade no município de Tomé-Açu, onde os dados obtidos corroboram para essa afirmação. Isto posto, os indicadores econômicos apresentaram dados positivos e satisfatórios ao produtor, haja vista que a partir da manipulação dos dados, os indicadores geraram os seguintes resultados: um VPL de $\mathrm{R} \$ 90.043,93$, um IBC no valor de R\$ 4,48, uma TIR de 77,74\% e um Ponto de Equilíbrio equivalente a $2.825 \mathrm{Kg}$, gerando um retorno do investimento em 2 anos e 3 meses, utilizando-se de um programa de financiamento com taxa de 10\%, atestando a disponibilidade do projeto em suportar a taxa mais alta considerada na pesquisa.

Logo, acentua-se que os mecanismos, técnicas e ainda a aplicação dos cálculos realizados nessa pesquisa não são absolutos, assim como, os conceitos, e o andamento da análise financeira, haja vista que cada região possui suas especificidades e adaptabilidade mercadológica, compreendendo que em cada território a demanda sofre variações. Deste modo, os resultados obtidos exprimem que o mercado de consumo do peixe na localidade é favorável, despertando o interesse de novos entrantes a atuarem na implantação dessa cultura, uma vez que, esse sistema é economicamente viável na região.

\section{REFERÊNCIAS}

ASSAF NETO, A.; LIMA, F. G.. Curso de Administração Financeira. São Paulo: Atlas, 2009.

BORDEAUX-RÊGO, R.; PAULO, G. P.; SPRITZER, I. M. P. A.; ZOTES, L. P.. Viabilidade econômico-financeira de projetos. 4 ed. Rio de Janeiro: FGV, 2013.

GIL, A. C.. Como elaborar projetos de pesquisa. 4 ed. São Paulo: Atlas, 2002.

MARTINS, C. V. B.; OLIVEIRA, D. P.; MARTINS, R. S.; HERMES C. A.; OLIVEIRA, L. G.; VAZ, S. K.; MINOZZO, M. G.; CUNHA, M.; ZACARKIN, C. E.. Avaliação da piscicultura na região Oeste do Estado do Paraná. Boletim do Instituto de Pesca, São Paulo, n.27, v.1, p.77-84, 2001.
NEVES, W. G.. Estudo da viabilidade econômica financeira para uma empresa de cosméticos. Monografia (Bacharelado em Administração) - Universidade Federal do Rio Grande do Sul, Porto Alegre, 2010.

SCHULTER, E. P.; VIEIRA FILHO, J. E. R.. Evolução da piscicultura no Brasil: diagnóstico e desenvolvimento da cadeia produtiva de tilápia. Rio de Janeiro: IPEA, 2017.

VILELA, M. C.; ARAÚJO, C. D.; MACHADO, L. S.; MACHADO M. R. R.. Análise da viabilidade econômico-financeira de projeto de piscicultura em tanques escavados. In: CONGRESSO USP DE INICIAÇÃO CIENTÍFICA EM CONTABILIDADE, 9. Anais. São Paulo: USP, 2012.

A CBPC - Companhia Brasileira de Produção Científica (CNPJ: 11.221.422/0001-03) detém os direitos materiais desta publicação. Os direitos referem-se à publicação do trabalho em qualquer parte do mundo, incluindo os direitos às renovações, expansões e disseminações da contribuição, bem como outros direitos subsidiários. Todos os trabalhos publicados eletronicamente poderão posteriormente ser publicados em coletâneas impressas sob coordenação da Sustenere Publishing, da Companhia Brasileira de Produção Científica e seus parceiros autorizados. Os (as) autores (as) preservam os direitos autorais, mas não têm permissão para a publicação da contribuição em outro meio, impresso ou digital, em português ou em tradução. 